\title{
Individual multi-catheter mould technique in high- dose-rate brachytherapy - personalized approach in treating multifocal angiosarcoma of the face
}

\author{
Marta Szlag, PhD!, Piotr Wojcieszek, PhD², Sylwia Kellas-Ṡleczka, PhD², Kamil Krysiak, MD³, Agnieszka Cholewka, Msc', \\ Małgorzata Stapór-Fudziniska, PhD!, Tomasz Krzysztofiak, MD², Marek Fijałkowski, MD² \\ IRadiotherapy Planning Department, Cancer Center and Institute of Oncology Gliwice Branch, Gliwice, Poland, ${ }^{2}$ Brachytherapy Department, \\ Cancer Center and Institute of Oncology Gliwice Branch, Gliwice, Poland, ${ }^{3}$ Radiotherapy Department, Cancer Center and Institute of \\ Oncology Gliwice Branch, Gliwice, Poland
}

\begin{abstract}
Purpose: The aim of this study was to report individual surface mould multi-catheter high-dose-rate (IMM HDR) application as a salvage treatment for a head and neck angiosarcoma patient, previously treated with surgery followed by external beam radiotherapy.

Material and methods: A 74-year-old male reported to our center with an uncommon malignant neoplasm of blood vessels. The patient was qualified for a wide local excision (WLE) of tumor with simultaneous reconstruction using a free-flap collected from the patient's thigh. After surgery, the patient was qualified for adjuvant external-beam radiotherapy (EBRT). Volumetric arc therapy (VMAT; RapidArc ${ }^{\circledR}$, Varian Medical Systems) was used to deliver $52.8 \mathrm{~Gy} / 1.6 \mathrm{~Gy}$ in 33 fractions. Overall treatment time was 51 days. Six months after radiotherapy, an incisional biopsy of non-healing ulcer of the nasal bridge revealed angiosarcoma. Wide local excision with skin graft reconstruction was planned. Due to multifocal disease and lack of possibility for further margin, the resection surgery was completed after skin graft reconstruction from the right thigh. Surface IMM HDR was considered as an alternative option for further treatment. Total dose of 48 Gy (12 fractions) was planned.

Results: One month after surface IMM HDR, healing process of the skin was observed in the treated regions and six months later, the irradiated areas recovered. General condition of the patient deteriorated two years after diagnosis and one year after HDR. He was hospitalized to receive palliative care.

Conclusions: HDR brachytherapy may be a valuable option for angiosarcoma treatment. Difficult lesion location may yield non-radical surgery. Surface IMM HDR provides highly conformal plan and allow adjusting the dose to individual clinical situation.

Key words: angiosarcoma, brachytherapy, individual mould applicator, skin cancer.

\section{Purpose}

Angiosarcoma incidence is $0.01 / 100,000$ or 1 in 10 million per year [1]. It is a rare neoplasm, and only $1-2 \%$ of all sarcomas are angiosarcomas [2,3,4]. More than $30 \%$ of patients present this skin tumor most often located in the head and neck region [5,6]. As an aggressive disease with poor prognosis, i.e., high-rate of distant and loco-regional metastases, it requires a multimodal approach. In order to achieve ultimate success in the angiosarcoma treatment is to perform radical surgery [2,7], which may be challenging for reaching uninvolved margins, especially in the head and neck region [8]. Adjuvant chemotherapy and radiotherapy are assumed to be a pri- mary treatment for tumor stage II and higher [9]. Another challenge is the management of failure after primary therapy $[8,9,10,11,12]$. One of the methods, which could be utilized, is surface high-dose-rate (HDR) brachytherapy, particularly in the head and neck region [13,14,15]. National Comprehensive Cancer Network (NCCN) guidelines for post-operatively management of soft tissue sarcoma of extremity, trunk, and head and neck region recommends delivery of $45 \mathrm{~Gy}$ with low-dose-rate (LDR) brachytherapy or HDR equivalent, with the status of surgical margins negative [9].

Surface HDR brachytherapy (BT) is one of the methods in the treatment of non-melanoma skin cancer $[16,17]$. Its efficacy has been established in the literature, but the

\footnotetext{
Address for correspondence: Marta Szlag, PhD, Cancer Center and Institute of Oncology Gliwice Branch, Received: 02.03.2019 15 Wybrzeże Armii Krajowej St., 44-102 Gliwice, Poland, phone: +48 3227892 53, 
use of this way of treatment in the head and neck angiosarcoma is poorly documented $[13,14,15]$.

There are two main techniques used in the surface brachytherapy [16,17]. Default applicators supplied by different companies worldwide can be used with convenience, but there are lesser options for treatment tailoring. Individual mould applicator is a choice for surface HDR in the problematic planning area (i.e., curved area, head and neck region, lesions located close to crucial organs at risk such as eye or brain) $[13,18,19]$. Individual mould multi-catheter HDR (IMM HDR) technique allows for precise adaptation of the dose coverage to the treated area [19]. Moreover, high HDR conformity, which includes steep dose gradient due to use of iridium-192 ( $\left.{ }^{192} \mathrm{Ir}\right)$ source and individually adjusted lead shielding, allows to escalate the dose in the target volumes with normal tissues sparing.

We aim to present surface IMM HDR applied as a salvage treatment for a patient with head and neck angiosarcoma previously treated with surgery followed by external beam radiotherapy.

\section{Material and methods}

\section{Initial treatment}

A 74-year-old male reported to our center with non-healing tumor mass on the left temple in 2015. It was $2 \mathrm{~cm}$ in the diameter with a history of 12 months. Biopsy of the lesion showed an uncommon malignant neoplasm of blood vessels, probably angiosarcoma. Immunophenotype was performed, and Ki67 was equal to 60\%, CD31 (+),
CD34 (-), F VIII (+/-), CK AE1/AE3 (-), HMB-45 (-), s-100 (-), and melan A (-). Computed tomography (CT) was used to determine the exact extent of the lesion. Despite the visible $2 \mathrm{~cm}$ ulcer, CT scan revealed that the tumor was $9 \mathrm{~mm}$ thick in the maximum and $7 \mathrm{~cm}$ wide in the largest diameter. Even though, it was positioned mostly in the temporal area, it spread to the borders of frontal and parietal areas. Additional ultrasound examination confirmed the extent of the abnormal tissue. The patient was qualified for a wide local excision (WLE) of tumor with simultaneous reconstruction using a free-flap collected from the patient's thigh. Positive margins were assessed intraoperatively. Margins were widened three times, and additional microscopic evaluation was done, with no success. Due to excision of dimensions ( $14 \mathrm{~cm}$ in the diameter) and probable orbital penetration, the surgery was completed after reconstruction as planned. The patient was revised on day 2. An intervention was needed because of the abnormal venous outflow from the flap. A local revision was also performed on day 5. The partial free-flap loss was observed; the injury was supplied with full-thickness skin graft collected from the right upper arm. Partial necrosis was observed on day 12; no intervention was applied. The patient was discharged on day 25 . Histopathological examination of the tissue resected during surgery reported cutaneous angiosarcoma (CD34 (+), CD31 (+), Ki67 20\%), with positive resection margins.

Two weeks later, the patient visited our outpatient Sarcoma and Bone Tumor Unit, and an adjuvant external beam radiotherapy (EBRT) was proposed. Treatment

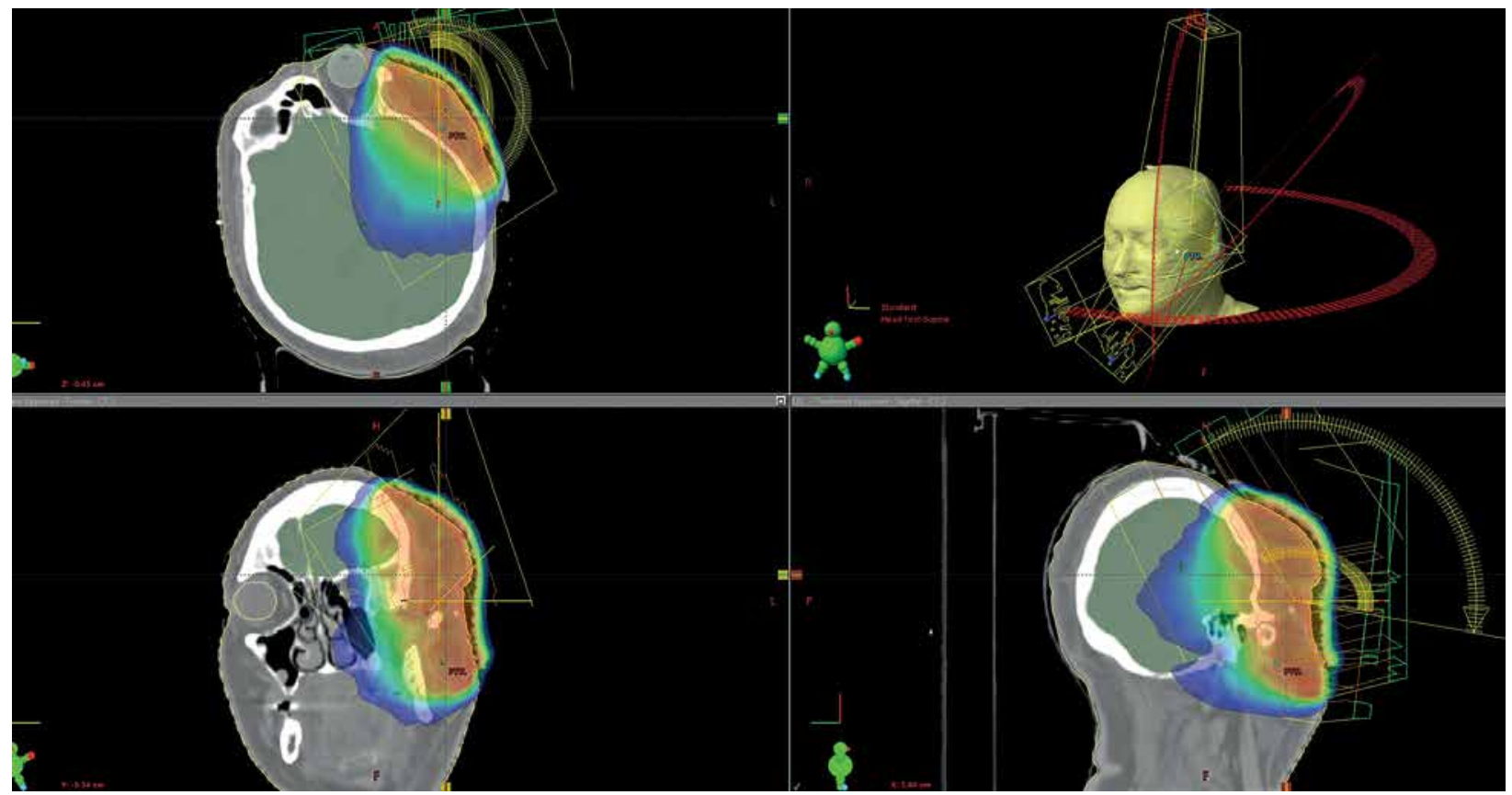

Fig. 1. Axial, coronal, and sagittal view of computed tomography (CT) with dose distribution presented in the dose-color-wash mode. 3D reconstruction presents patient body outline and beam trajectory. Red line represents gross tumor volume (GTV) and orange lines represent clinical target volume (CTV). Organs at risk (OARs) were contoured for treatment planning and plan optimization. The treatment plan was calculated with EclipseTM planning system (Varian Medical System) with AAA v. 10.0.28 (Anisotropic Analytical Algorithm). Calculation dose grid was $2.5 \times 2.5 \times 2.0 \mathrm{~mm}$. VMAT technique was used for plan development and realization. $6 \mathrm{MV}$ photon beams and beam intensity modulation was used to deliver 52.8 Gy in 33 fractions to PTV. The overall treatment time was 51 days 
planning started after individual consent. The dose schedule was chosen according to our Radiotherapy Department guidelines for adjuvant radiotherapy in freeflap reconstructed area. Volumetric arc therapy (VMAT; RapidArc ${ }^{\circledR}$, Varian Medical Systems) was used to deliver of 52.8 Gy/1.6 Gy in 33 fractions (dose intensity modulation, $6 \mathrm{MV}$ photon beams, Figure 1). The overall treatment time lasted for 51 days.

The total EBRT dose was reduced to decrease the toxicity risk in the free-flap region located in the proximity of radiation areas. Dose distribution was calculated according to dose constraints of our Radiotherapy Department with free-flap region treatment protocol. The patient was referred to the outpatient clinic for follow-up.

First CT scan was performed three months after adjuvant radiotherapy. The reconstructed area was recovered with no recurrence. Three months later $(6$ months after radiotherapy), the patient reported to a surgeon with a non-healing ulcer of the nasal bridge. Incisional biopsy revealed angiosarcoma. Wide local excision with skin graft reconstruction was planned. An intraoperative histopathologic report showed positive margins and another angiosarcoma spot close to left eyebrow. Due to multifocal disease and lack of possibility for further margin resection, the surgery was completed after skin graft reconstruction from right thigh.

The patient was not qualified for the second external beam radiotherapy, because the residual tumor was located too close to an eye, an area that was previously reconstructed and irradiated. Two months after the second surgery, the patient was referred to our Brachytherapy Department. Even though there was no data, surface IMM HDR was considered as an alternative option.

\section{Brachytherapy treatment}

On the baseline, the patient had a visual impairment in the left eye. Moreover, erythema of both left eyelids was reported with sclera injection and skin necrosis located close to the medial angle of the left eye. There were two main angiosarcoma foci on the face. The first one was localized on the right side from right nasolabial fold to the right medial eye angle, and the second included the left temple, left upper eyelid, and left nasolabial fold. The disease progressed rapidly as both lesions were fast-growing.

Due to lesions expansion and localization on both sides of the face, the treatment was split into two stages. Brachytherapy was performed with two individually designed custom moulds. These are made, based on the individual local imprint, with dental impression silicone covering visible tumor mass on the skin surface with $1 \mathrm{~cm}$ margin. The surface reflection is done in the plaster model to press the acrylic plate on it and to gain an individual mould ( $3 \mathrm{~mm}$ thick). Catheters (plastic tubes) are fixed to the opposite side of anatomic surface. The number and spatial configuration of the catheters are individually adjusted to achieve optimal conformity and homogeneity of the dose distribution and target coverage.

Dose distribution delivered with IMM HDR brachytherapy is a leading method for delivering high-dose to the target located on the surface of the skin, with simultaneous sparing of healthy tissue located below the treated region. In comparison to EBRT, relative dose delivered to the target is high, while steep dose gradient allows for reducing the dose in the healthy structures (Figure 2).

CTV1 was designated for the right side, while CTV2 included left part of the face. Target volumes were specified based on the tumor mass observed on the patient skin. A margin for sub-clinical disease spread was added to the target volumes. According to the rules included in our internal, departmental protocol for non-melanoma skin cancer, clinical target volume (CTV) is a $10 \mathrm{~mm}$ margin added circumferentially to the target volume. CTV1 and CTV2 were planned to be treated separately with

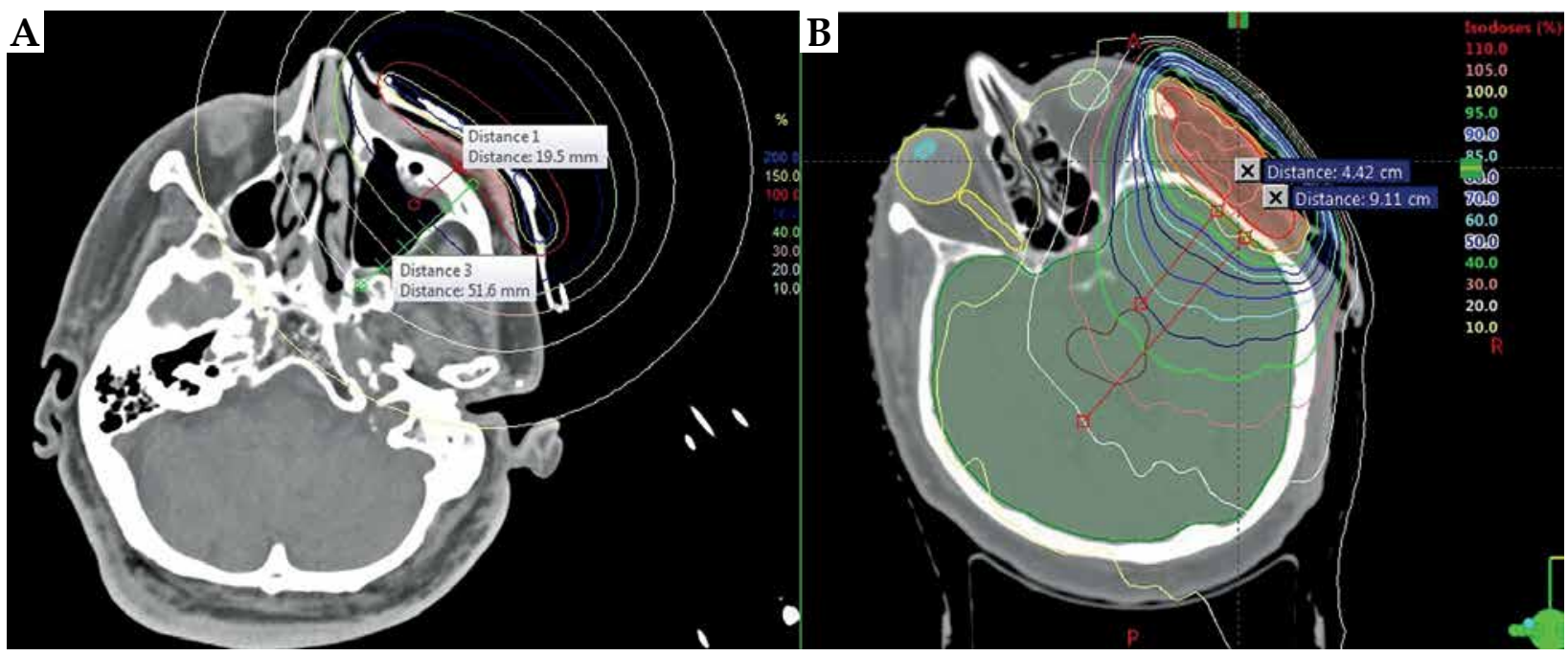

Fig. 2. Treatment plan calculated for (A) CTV2 (brachytherapy) and (B) PTV (EBRT). Dose distribution is presented in a relative mode as a percentage of the prescribed dose. Relative dose gradient was measured and compared between plans. Dose gradient was measured from the $100 \%$ to the $50 \%$ isodose and $20 \%$ isodose on the selected plane. Distance between isodoses differ for BT and EBRT treatment plan 
one-week gap. Two moulds were prepared. Eleven and twenty-one catheters were used for CTV1 and CTV2, respectively.

OncentraBrachy treatment planning system v. 4.5.2 (Elekta Instrument AB Stockholm) was used for planning 3D dose distribution. The dose was calculated on $\mathrm{CT}$, with $1 \mathrm{~mm}$ slice thickness. The reference dose was initially specified as $5 \mathrm{~mm}$ (average) from the individual mould surface for both CTV1 and CTV2 (Figure 3). In our department, for contact brachytherapy, the reference dose is delivered according to rules included in departmental protocol, which was established based on the 20-year experience in treating non-melanoma skin cancer with superficial applicators. The $5 \mathrm{~mm}$ dose specification from the surface of the applicator (below the patient skin surface) has been recognized as a safe and efficient method of treating superficially located lesions. 3D treatment planning based on CT allows for CTV contouring and dose prescription based on the specified target volume. Introducing 3D treatment planning into clinical practice is a complex process. To fully benefit from 3D imaging, a clinical results collected from the 2D era should be considered as a starting point for prescribing and optimizing 3D dose distribution. Therefore, the reference dose was specified as $5 \mathrm{~mm}$ below the surface of the applicator, and then the optimization of dwell times was applied to improve CTV coverage in the region where the tumor penetrated deeper (more than $5 \mathrm{~mm}$ ) into the tissue, but the dose of $200 \%$ of the reference dose should not exceed the maximal skin dose. Maximal dose point of $200 \%$ was the dose constraint for the skin. The depth and the range of the reference isodose was also compromised by the normal tissues as well as eyes and previously treated region located in the proximity of CTV1 and CTV2.

The total dose of $48 \mathrm{~Gy}$ (reference dose) in 12 fractions was planned. Four fractions per week were scheduled for both CTVs to three weeks of overall treatment time per side. The dose was specified on $100 \%$ isodose. The total dose was compromised by the dose delivered previously with external beam therapy and the extent of the lesion. Eye shielding for reducing the dose delivered to both lenses was used; round plates of lead ( $3 \mathrm{~mm}$ thick) were placed on the patient eyelids at every fraction.

\section{Results}

One month after surface IMM HDR, healing process of the skin was observed in treated regions. Skin erythema with diffused inflammation and desquamation was reported. Moreover, acute erythema of left eyelid with severe conjunctivitis including corneal drying and scleral atrophy was diagnosed by ophthalmologist, probably both after EBRT and HDR. In the next three months of the follow-up, the vision in the left eye improved (patient reacted to light), but conjunctivitis, corneal drying, and scleral atrophy were as before. Six months from HDR, skin erythema was still observed, but irradiated areas recovered (Figure 4). During the next eight months of follow-up, purulent dermatitis in the left nasolabial fold was diagnosed and cured in our center's surgical ambulatory. The general condition of the patient has worsened two years after diagnosis and one year after HDR. Local
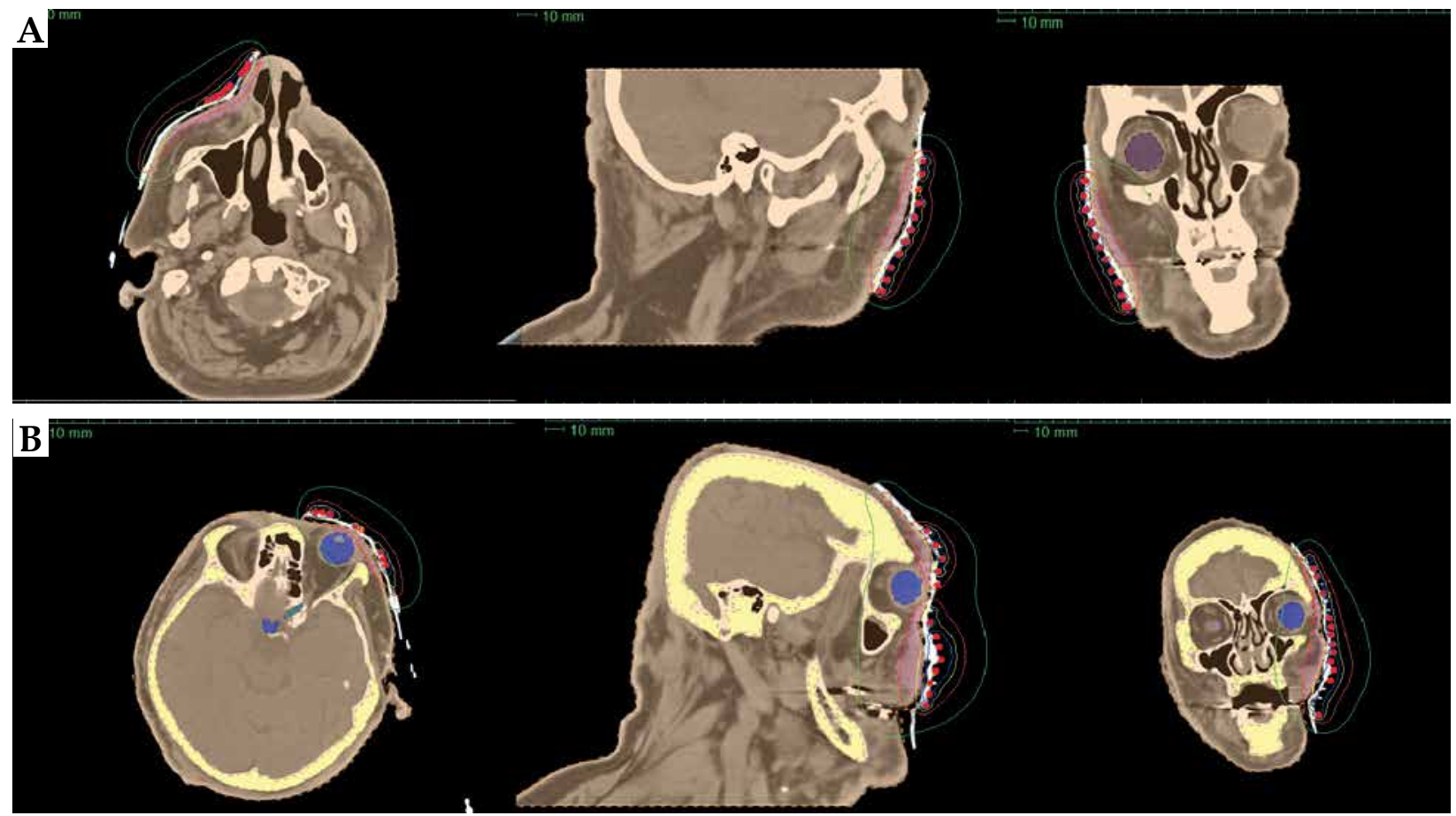

Fig. 3. Axial, coronal, and sagittal view of computed tomography (CT)-based treatment plan for CTV1 (A) and CTV2 (B). Contour of the patient body together with the reference isodose line (red line) and reconstructed catheters. Treatment plans were calculated with OncentraMasterPlan v. 4.5.2. Dose distribution was calculated based on the axial CT scans acquired with $1 \mathrm{~mm}$ thickness 

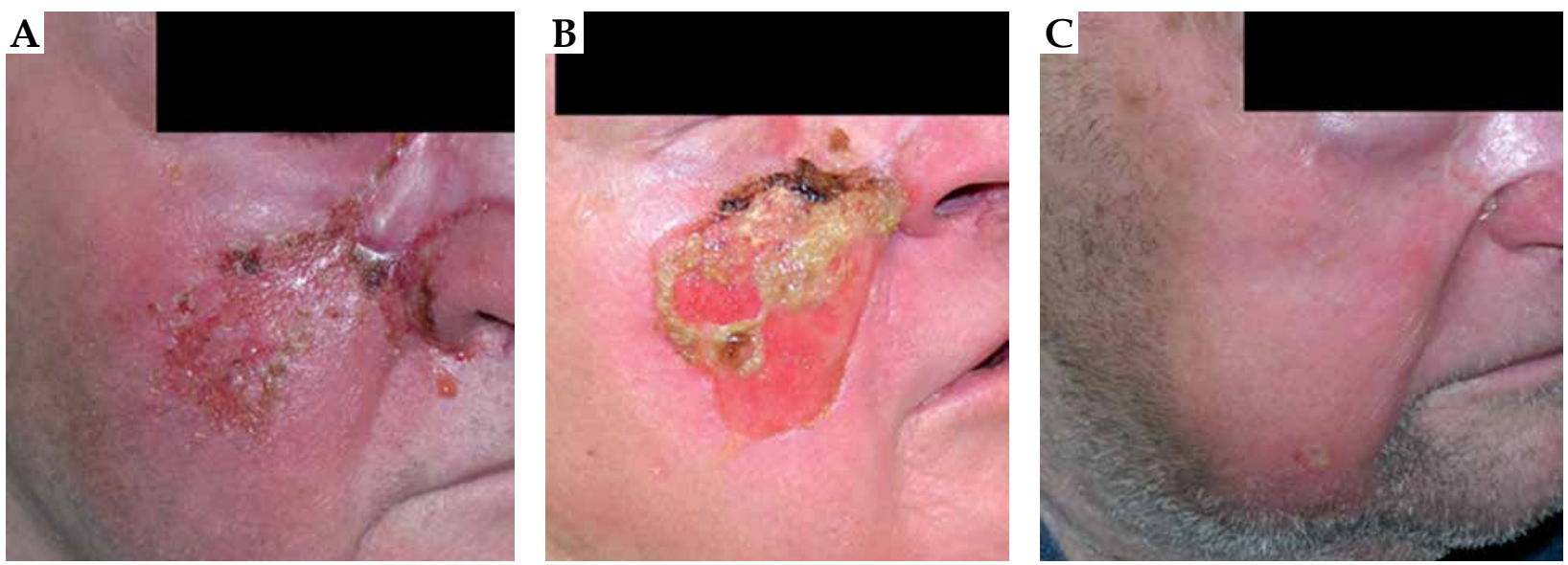

Fig. 4. Skin of the nasolabial fold on the right side of the face (A) before the brachytherapy, (B) at twelfth brachytherapy fraction, and $(\mathbf{C})$ three months after treatment

recurrence had occurred 11/12 months after finishing the treatment. The patient was hospitalized to receive palliative care.

\section{Discussion}

Angiosarcoma accounts for no more than $2 \%$ of all soft tissue sarcomas, which is a very complex and heterogeneous group of tumors [2,3]. Survival rates of cutaneous angiosarcoma correlate with age, multifocal disease, anatomical site, and stage of disease [5]. Based on the analysis of the group of 50 patients, Bernstein et al. demonstrated worse survival of scalp angiosarcoma than face angiosarcoma [6]. Most of the published data on angiosarcoma treatment show small groups of patients collected throughout the decades $[8,20,21]$. Treatment procedures including surgery and radiotherapy have evolved in the last decades. Irradiation techniques used for the treatment of angiosarcoma have changed significantly, which may have an impact on the treatment results. Although published data on brachytherapy for angiosarcoma are mostly case reports, every individually approached patient and results reported are valuable because of the rarity of this disease $[2,13,15,22,23]$.

In our case, high-dose-rate brachytherapy was used to treat multifocal angiosarcoma located on the face. The main advantage of this treatment was the possibility of the dose distribution tailoring due to an individual multi-catheter mould used. IMM HDR allowed optimizing dwell time and position of the source to deliver the optimal dose in the problematic locations (curved surface or proximity of the organs at risk). Moreover, the extent of the disease and its fast progression made the treatment complicated. The treatment was delivered with salvage intent, and it was achieved as a rapid decrease of the lesions and an acceptable cosmetic result shortly after brachytherapy. Additionally, patient's psychological condition strengthened after treatment apart from left eye vision improvement.

Efficacy and safety of the surface brachytherapy in the skin lesions management, especially non-melanoma skin cancer, has been well documented. Different tech- niques and applicators can be used to deliver a dose to skin $[16,17,24,25]$. Stepping source $\left({ }^{192} \mathrm{Ir}\right)$ has an optimal dose profile to deliver the dose to the skin surface efficiently with excellent and good cosmetic outcomes [24]. IMM HDR seems to be an optimal approach to treat lesions in the problematic areas, particularly in the head and neck region. Unfortunately, there is scarce data on surface HDR in the angiosarcoma patients, probably because angiosarcoma is a rare condition with an aggressive history $[13,14,15]$. The overall prognosis of head and neck angiosarcoma is poor [7,14,26,27]. Patel et al. analyzed retrospectively 56 patients with scalp/face angiosarcomas treated in Mayo Clinic [27]. Eighty per cent of patients underwent surgery as a wide local excision \pm reconstruction. Although, radical resection including reresection was planned, final histopathologic examination revealed positive margins in $12(27 \%)$ patients. Majority of patients $(65 \%)$ received adjuvant treatment (i.e., radiotherapy, chemotherapy or both). Other patients received single treatment $(28 \%)$ or combination of radio- and chemotherapy (7\%). Median follow-up was 25 months, with $38 \%$ and $16 \%$ of overall (OS) and recurrence-free survival (RFS) at 5 years, respectively. Univariate analysis showed survival benefit of $46 \%$ for combined modality therapy. Ogawa et al. analyzed failure patterns in the group of 48 patients suffering from head and neck angiosarcoma [3]. Median survival time was 13.4 months. Their data showed that combination of surgery and radiotherapy had a significantly better prognosis than sole surgery/ radiotherapy or no surgery/no radiotherapy approaches (2-year OS: $46 \%$ vs. $11 \%$ vs. $0 \%$; $p<0.0001$ ). Sanada et al. presented a group of 9 patients with head and neck angiosarcoma treated with image-guided brachytherapy [14]. Only two patients received surgery before irradiation and seven received chemo- or immunotherapy. The 3 -year OS and local control were $51 \%$ and $78 \%$, respectively. Significant improvement in the local control was reported for patients treated with brachytherapy doses higher than $60 \mathrm{~Gy}$ in 20 fractions.

Wide local excision with negative margins is the treatment of choice for angiosarcoma $[8,28,29,30]$, even though it may not be reachable due to an extensive subclinical 
tumor spread [8]. Adjuvant irradiation plays an important role in enhancing local control and overall survival. Combination of these two modalities is the most efficient way of treatment $[6,8,11,12,14,23,26,27,28,30]$. Due to its advantages, HDR should be considered as one of the useful techniques in angiosarcoma management, while IMM HDR could be beneficial in the individualized approach, especially with problematic treatment area. The latest NCCN guidelines for soft tissue sarcoma consider brachytherapy as an equivalent technique to external beam therapy [9].

\section{Conclusions}

High-dose-rate brachytherapy may be a valuable option for angiosarcoma treatment in cases where difficult lesion location may yield non-radical surgery (i.e., positive margins). Surface IMM HDR provides high conformity in respect to EBRT treatment. Catheters, which are individually placed in the mould, allow optimizing the dose to shape its coverage to anatomic structures and individual clinical situation. It enhances the dose inside target volumes and decrease the dose delivered to the organs at risk (OARs).

\section{Disclosure}

Authors report no conflict of interest.

\section{References}

1. Kohlmeyer J, Steimle-Grauer SA, Hein R. Cutaneous sarcomas. J Dtsch Dermatol Ges 2017; 15: 630-648.

2. Ishida Y, Otsuka A, Kabashima K. Cutaneous angiosarcoma: update on biology and latest treatment. Curr Opin Oncol 2018; 30: 107-112.

3. Ogawa K, Takahashi K, Asato Y et al. Treatment and prognosis of angiosarcoma of the scalp and face: a retrospective analysis of 48 patients. Br J Radiol 2012; 85: e1127-1133.

4. Glickstein J, Sebelik Me, Lu Q. Cutaneous angiosarcoma of the head and neck: a case presentation and review of the literature. Ear Nose Throat J 2006; 85: 672-674.

5. Albores-Saavedra J, Schwartz AM, Henson DE et al. Cutaneous angiosarcoma. Analysis of 434 cases from the Surveillance, Epidemiology, and End Results Program, 1973-2007. Ann Diagn Pathol 2011; 15: 93-97.

6. Bernstein JM, Irish JC, Brown DH et al. Survival outcomes for cutaneous angiosarcoma of the scalp versus face. Head Neck 2017; 39: 1205-1211.

7. Lee KC, Chuang SK, Philipone EM, Peters SM. Characteristics and prognosis of primary head and neck angiosarcomas: a Surveillance, Epidemiology, and End Results Program (SEER) analysis of 1250 cases. Head Neck Pathol 2018.

8. Requena C, Sendra E, Llombart B et al. Cutaneous angiosarcoma: clinical and pathology study of 16 cases. Actas Dermosifiliogr 2017; 108: 457-465.

9. NCCN Clinical Practice Guidelines in Oncology (NCCN Guidelines ${ }^{\circledR}$ ) Soft Tissue Sarcoma. Version 2.2019, February 4, 2019.

10. Dossett LA, Harrington M, Cruse CW, Gonzalez RJ. Cutaneous angiosarcoma. Curr Probl Cancer 2015; 39: 258-263.

11. Bedford JL, Childs PJ, Hansen VN et al. Treatment of extensive scalp lesions with segmental intensity-modulated photon therapy. Int J Radiat Oncol Biol Phys 2005; 62: 1549-1558.
12. Guadagnolo BA, Zagars GK, Araujo D et al. Outcomes after definitive treatment for cutaneous angiosarcoma of the face and scalp. Head Neck 2011; 33: 661-667.

13. Nakamura R, Harada S, Obara T et al. Iridium-192 brachytherapy for hemorrhagic angiosarcoma of the scalp: a case report. Jpn J Clin Oncol 2003; 33: 198-201.

14. Sanada T, Nakayama H, Irisawa R et al. Clinical outcome and dose volume evaluation in patients who undergo brachytherapy for angiosarcoma of the scalp and face. Mol Clin Oncol 2017; 6: 334-340.

15. Wittych J, Banatkiewicz P, Wachowicz M et al. Angiosarcoma of the scalp: a case report. J Contemp Brachytherapy 2014; 6: 208-212.

16. Guinot JL, Rembielak A, Perez-Calatayud J et al. GECESTRO ACROP recommendations in skin brachytherapy. Radiother Oncol 2018; 126: 377-385.

17. Skowronek J. Brachytherapy in the treatment of skin cancer: an overview. Postepy Dermatol Alergol 2015; 32: 362-367.

18. Kowalik L, Lyczek J, Sawicki M, Kazalski D. Individual applicator for brachytherapy for various sites of superficial malignant lesions. J Contemp Brachytherapy 2013; 5: 45-49.

19. Kuncman L, Kozlowski S, Pietraszek A et al. Highly conformal CT based surface mould brachytherapy for non-melanoma skin cancers of earlobe and nose. J Contemp Brachytherapy 2016; 8: 195-200.

20. Hodgkinson DJ, Soule EH, Woods JE. Cutaneous angiosarcoma of the head and neck. Cancer 1979; 44: 1106-1113.

21. Miki Y, Tada T, Kamo R et al. Single institutional experience of the treatment of angiosarcoma of the face and scalp. $\mathrm{Br}$ J Radiol 2013; 86: 20130439.

22. Patel VB, Speer TW. Successful treatment of an angiosarcoma of the nose with radiation therapy. Case Rep Oncol 2012; 5: 570-575.

23. Tenjarla S, Sheils LA, Kwiatkowski TM, Chawla S. Cutaneous angiosarcoma of the foot: a case report and review of the literature. Case Rep Oncol Med 2014; 2014: 657876.

24. Delishaj D, Rembielak A, Manfredi B et al. Non-melanoma skin cancer treated with high-dose-rate brachytherapy: a review of literature. J Contemp Brachytherapy 2016; 8: 533-540.

25. Rosen EB, Ko E, Wolden $\mathrm{S}$ et al. Intraoral angiosarcoma: treatment with a brachytherapy prosthesis. J Prosthet Dent 2015; 113: 242-245.

26. Mark RJ, Tran LM, Sercarz J et al. Angiosarcoma of the head and neck. The UCLA experience 1955 through 1990. Arch Otolaryngol Head Neck Surg 1993; 119: 973-978.

27. Patel SH, Hayden RE, Hinni ML et al. Angiosarcoma of the scalp and face: the Mayo Clinic experience. JAMA Otolaryngol Head Neck Surg 2015; 141: 335-340.

28. Abraham JA, Hornicek FJ, Kaufman AM et al. Treatment and outcome of 82 patients with angiosarcoma. Ann Surg Oncol 2007; 14: 1953-1967.

29. Holden CA, Spittle MF, Jones EW. Angiosarcoma of the face and scalp, prognosis and treatment. Cancer 1987; 59: 10461057.

30. Pawlik TM, Paulino AF, McGinn CJ et al. Cutaneous angiosarcoma of the scalp: a multidisciplinary approach. Cancer 2003; 98: 1716-1726. 